\title{
Low skeletal muscle area is a risk factor for mortality in mechanically ventilated critically ill patients
}

Peter JM Weijs ${ }^{1,2,3^{*}}$, Wilhelmus GPM Looijaard ${ }^{2}$, Ingeborg M Dekker ${ }^{1}$, Sandra N Stapel ${ }^{2}$, Armand R Girbes ${ }^{2,5}$, Heleen M Oudemans-van Straaten ${ }^{2,5}$ and Albertus Beishuizen ${ }^{2,4,5}$

\begin{abstract}
Introduction: Higher body mass index (BMI) is associated with lower mortality in mechanically ventilated critically ill patients. However, it is yet unclear which body component is responsible for this relationship.

Methods: This retrospective analysis in 240 mechanically ventilated critically ill patients included adult patients in whom a computed tomography (CT) scan of the abdomen was made on clinical indication between 1 day before and 4 days after admission to the intensive care unit. CT scans were analyzed at the L3 level for skeletal muscle area, expressed as square centimeters. Cutoff values were defined by receiver operating characteristic (ROC) curve analysis: $110 \mathrm{~cm}^{2}$ for females and $170 \mathrm{~cm}^{2}$ for males. Backward stepwise regression analysis was used to evaluate low-muscle area in relation to hospital mortality, with low-muscle area, sex, BMl, Acute Physiologic and Chronic Health Evaluation (APACHE) II score, and diagnosis category as independent variables.

Results: This study included 240 patients, 94 female and 146 male patients. Mean age was 57 years; mean BMI, $25.6 \mathrm{~kg} / \mathrm{m}^{2}$. Muscle area for females was significantly lower than that for males $\left(102 \pm 23 \mathrm{~cm}^{2}\right.$ versus $158 \pm 33 \mathrm{~cm}^{2}$; $P<0.001$ ). Low-muscle area was observed in $63 \%$ of patients for both females and males. Mortality was $29 \%$, significantly higher in females than in males (37\% versus $23 \% ; P=0.028)$. Low-muscle area was associated with higher mortality compared with normal-muscle area in females $(47.5 \%$ versus $20 \% ; P=0.008)$ and in males (32.3\% versus $7.5 \% ; P<0.001$ ). Independent predictive factors for mortality were low-muscle area, sex, and APACHE II score, whereas BMI and admission diagnosis were not. Odds ratio for low-muscle area was 4.3 (95\% confidence interval, 2.0 to $9.0, P<0.001)$. When applying sex-specific cutoffs to all patients, muscle mass appeared as primary predictor, not sex.

Conclusions: Low skeletal muscle area, as assessed by CT scan during the early stage of critical illness, is a risk factor for mortality in mechanically ventilated critically ill patients, independent of sex and APACHE II score. Further analysis suggests muscle mass as primary predictor, not sex. BMI is not an independent predictor of mortality when muscle area is accounted for.
\end{abstract}

\section{Introduction}

Patients admitted to the intensive care unit (ICU) are often severely ill, and many have muscle (protein) catabolism, muscle weakness, and/or atrophy, all linked to an increased morbidity and mortality [1]. Patients with inadequate energy and protein intake are at risk for

\footnotetext{
*Correspondence: p.weijs@vumc.nl

${ }^{1}$ Department of Nutrition and Dietetics, Internal Medicine, VU University

Medical Center Amsterdam, De Boelelaan 1117, Amsterdam, The Netherlands

${ }^{2}$ Department of Intensive Care Medicine, VU University Medical Center

Amsterdam, De Boelelaan 1117, Amsterdam, The Netherlands

Full list of author information is available at the end of the article
}

complications, including infections, acute respiratory distress syndrome, need for surgery, and renal failure [2,3]. Adequate reserves of body protein and fat mass at admission to the ICU may be crucial to recovery and survival.

A number of meta-analyses $[4,5]$ have been published on the observation of a lower hospital mortality in critically ill patients with a body mass index (BMI) in the overweight (BMI, 25 to 30) and obese (30 to 40) range [6]. Additionally, more recent studies [7-9] have reestablished these findings. This so-called obesity paradox is also observed in chronic conditions such as hemodialysis. 
Increasing evidence points to preserved muscle protein as part of the explanation [10].

Although BMI can easily be assessed in very large cohorts, only limited observations are available for actual and reliable measurement of protein mass. Recently, Baracos et al. developed a technique for muscle mass assessment by analyzing computed tomography (CT) scans in cancer patients $[11,12]$. They showed that sarcopenic obese patients, defined by a high BMI and a low muscle mass, have a higher mortality $[13,14]$. This observation suggests that "fat is good, but muscle is better." High BMI is not only associated with an increased fat mass, but also with an increased fat-free mass [15]. At the same time, a normal BMI is not exclusively based on a normal fat mass, but may be the result of a high fat mass with a relatively low muscle mass. Assessment of muscle mass might help to optimize protein and energy balance and improve risk assessment in the ICU [16].

Therefore, we investigated the relation between muscle mass as assessed from CT scans, as well as BMI, and outcome in a cohort of mechanically ventilated critically ill patients.

\section{Materials and methods Patients}

This is a retrospective analysis in a mixed medicalsurgical group of patients admitted to the ICU of a university hospital in the period $12 / 2003$ to $09 / 2012$. Inclusion criteria were age of 18 years or older, ICU stay of at least 4 days, mechanical ventilation during ICU stay, and an "early" CT scan of the abdomen (including L3 slice) made within a time frame of 1 day before admission to 4 days after admission for diagnostic and/ or interventional reasons. Exclusion criteria were CT scans not meeting quality checks (artifacts and muscle outside scanned frame) and missing data on body weight and height.

Patients eligible for this study were identified by searching the patient data-management system (PDMS) for patients who fulfilled the inclusion criteria and had the words "CT" and/or "scan" (excluding "bladder scan") in the nursing-notes section.

Demographic and clinical data including sex, age, weight, height, BMI, Acute Physiologic and Chronic Health Evaluation (APACHE) II score, admission diagnosis, length of stay (LOS) in the ICU and hospital, as well as ICU-, 28-day-, and hospital mortality were obtained from the PDMS (Metavision; IMDsoft, Tel-Aviv, Israel) and hospital information system (Mirador; iSOFT Nederland BV, Leiden, The Netherlands). The study was approved by the Institutional Review Board of the VU University Medical Center. The need for informed consent was waived because of the retrospective nature of the study using coded data obtained from routine care.

\section{CT scan analysis}

CT scans were imported from the local radiology system and assessed for quality. First, the third lumbar vertebra (L3) was identified on abdomen CT scans. This landmark was chosen because of its established correlation with whole-body muscle mass [17-19]. The L3 slice provides information on a number of muscles: the erector spinae-, quadratus lumborum-, psoas-, transversus abdominis-, interior- and exterior oblique-, and rectus abdominis muscles. The L3 slice was isolated and stored for later analysis.

CT scan analysis was performed by using Slice-Omatic version 4.3 and 5.0 (TomoVision, Montreal, QC, Canada) by trained personnel. Muscle tissue was identified by using boundaries in Hounsfield Units set to -29 to +150 [18]. The software computed a muscle surface area in $\mathrm{cm}^{2}$ by multiplying the pixel area by the amount of pixels identified as muscle.

BMI categories were used as defined by the World Health Organization [6].

\section{Statistics}

Fisher Exact- and $\chi^{2}$ tests were used to compare categoric variables and Mann-Whitney $U$ and $T$ tests for continuous variables not normally distributed, and $t$ test for continuous variables with a normal distribution.

ROC curve analysis was used to define muscle-area cutoff values best fit to predict hospital mortality in female and male patients separately.

Backward stepwise (Wald) regression analyses were performed, with hospital mortality as outcome variable. Independent variables were low muscle area (below sexspecific cutoff, $y / n)$, sex, BMI, APACHE II score, and admission diagnosis. Additionally, Kaplan-Meier curve analysis was performed to show the relation between the different muscle-area groups and mortality.

We also investigated muscle area as a continuous variable (expressed per $10 \mathrm{~cm}^{2}$ ) with stepwise logistic analysis. Last, another model was investigated by using the cutoff value for females for males as well, additional to the cutoff values for males. In this way, high-, medium-, and low-muscle area groups were created. SPSS 20 (SPSS Inc., Chicago, IL, USA) was used for statistical analysis. A $P<0.05$ was considered statistically significant.

\section{Results}

Figure 1 shows the consort diagram.

During the study period, 12,507 patients were admitted to the ICU with a mean APACHE II score of 17.44 (SD, 8.45). Of these, $852(6.8 \%)$ patients fulfilled the inclusion criteria, and CT scans were retrieved of 293 patients. Quality check of the CT scans revealed that 19 scans did not display all required muscles, 24 scans contained artifacts, and in 10 cases, the quality of the scan 


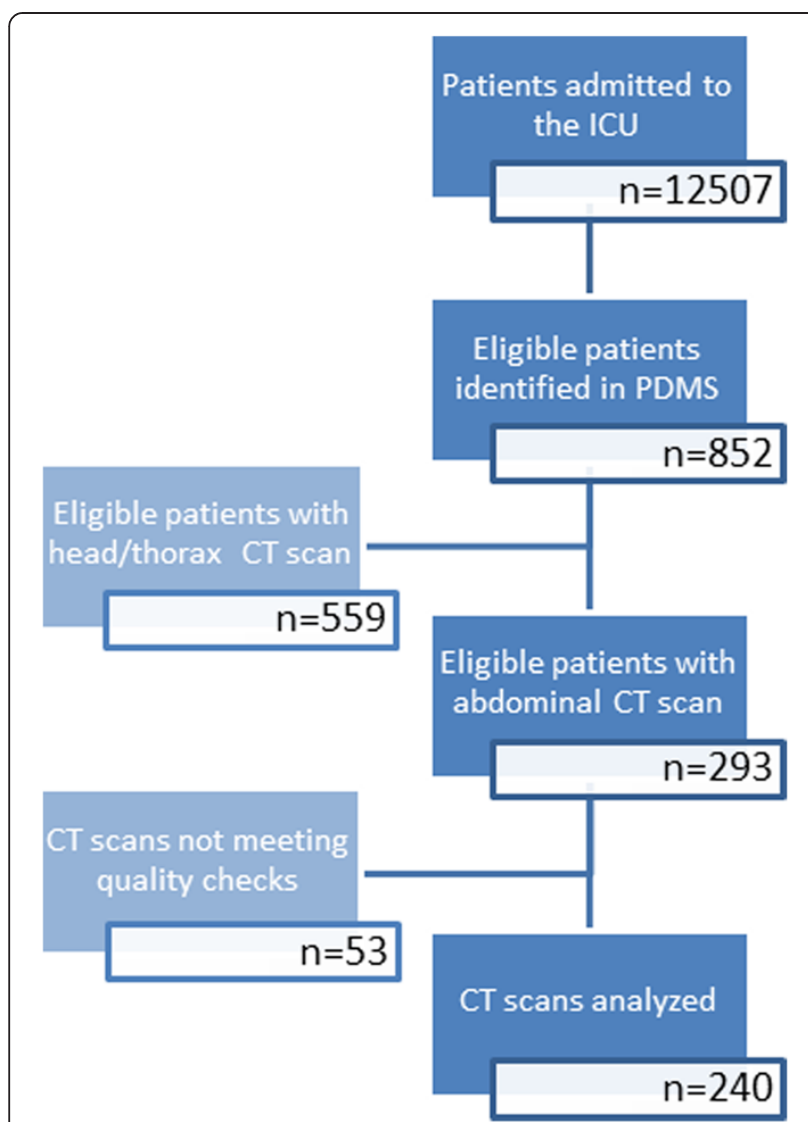

Figure 1 Consort diagram showing the inclusion process.

was considered insufficient for analysis. Therefore, 53 scans were excluded, and scans of 240 patients remained for further analysis. The study population therefore represents $35.5 \%$ of the patients fulfilling the inclusion criteria (ventilated patients with an ICU stay of at least 4 days) and $82 \%$ of the patients with an early CT scan. Mean APACHE II score of the study population was 23.4 (SD, 8.1). The mean interval between ICU admission and the CT scan was 0.74 (SD, 1.18) days.

Baseline characteristics of all patients, and of those with low and normal muscle area separately, are presented in Table 1; 240 patients were included (94 F, $146 \mathrm{M})$. Mean muscle area for females was lower than for males $\left(102 \pm 23 \mathrm{~cm}^{2}\right.$ versus $\left.158 \pm 33 \mathrm{~cm}^{2} ; P<0.001\right)$. We therefore determined cutoff values for low-muscle area for males and females separately. ROC curve analysis provided cutoff values of $110 \mathrm{~cm}^{2}$ for females and $170 \mathrm{~cm}^{2}$ for males. A low muscle area was observed in $63 \%$ of patients for both females (below $110 \mathrm{~cm}^{2}$ ) and males (below $170 \mathrm{~cm}^{2}$ ). Mortality was $29 \%$ and higher in females than in males (37\% versus $23 \% ; P=0.028$ ). Patients with low muscle area had a higher hospital mortality (38.2\% versus $12.5 \% ; P<0.001)$, in females $(47.5 \%$ versus $20.0 \%, P=0.008)$ as well as in males $(32.3 \%$ versus $7.5 \%$;
$P<0.001)$. Figure 2 shows the difference in mortality as Kaplan-Meier curves for the combined (females and males) low-muscle area group versus normal muscle-area group.

Stepwise regression analysis showed that muscle area, sex, and APACHE II score were independent predictors for hospital mortality, whereas BMI and admission diagnosis were not. Odds ratio for low-muscle area was 4.3 (95\% CI, 2.0 to 9.0; $P<0.001$ ). Table 2 shows results for three models, first the model with muscle area as a continuous variable, second for sex-specific cutoff values, and third for sex-combined cutoff values. In the sexcombined approach, creating low-medium-high muscle area groups, the low-muscle area group $\left(<110 \mathrm{~cm}^{2}\right)$ contains $63 \%$ of females and $8 \%$ of males, with similar hospital mortality rate ( $47.5 \%$ for females, $45.5 \%$ for males).

\section{Discussion}

This study shows that low skeletal muscle mass is an independent risk factor for mortality in mechanically ventilated critically ill patients. This observation is independent of sex and APACHE II score. The study also shows that when muscle area is accounted for, BMI appears to have no impact on mortality. The study group represents a more severely ill population still present in the ICU at day 4. Skeletal muscle mass was obtained from CT scans made for clinical reasons during the first days of ICU admission and expressed as skeletal muscle area at the level of the third lumbar vertebra.

Our findings are in line with a recent study by Moisey et al. [20] in 149 injured elderly ICU patients. They also found that low muscle mass, as assessed by CT scans, was associated with mortality. Our observations are made in an ICU representative age group, and not in an elderly population, as in Moisey's population. This study further adds new ICU-specific cutoff values. When the cutoff value for females was also applied for males (right panel in Table 2), a very high-risk group became apparent, of which one of two patients died during hospital stay. Reason for the higher mortality in our study with respect to age is that our study group represents a more severely ill population still present in the ICU at day 4, and Moisey took CT scans on admission [20].

Several studies have shown a relation between high BMI and lower mortality in ICU patients $[4,5,7,8]$, but results are not consistent [21]. However, a recent very large observational cohort study in 154.308 patients provided more evidence on this so-called obesity paradox [9]. The study found that overweight and obese patients with BMI up to 40 had the lowest risk of death. The authors argued that fat mass may play an important role in the immunologic response of the patient under stressed conditions in the ICU. However, our study shows that muscle area is a stronger predictor than BMI. BMI is 
Table 1 Patient characteristics and outcome for normal- and low-muscle area patient group

\begin{tabular}{|c|c|c|c|c|c|c|c|}
\hline & \multicolumn{2}{|c|}{$\begin{array}{l}\text { Normal muscle-area patients } \\
\qquad n=88\end{array}$} & \multicolumn{2}{|c|}{$\begin{array}{l}\text { Low muscle-area patients } \\
\qquad n=152\end{array}$} & \multicolumn{2}{|c|}{$\begin{array}{l}\text { All patients } \\
N=240\end{array}$} & \multirow[t]{2}{*}{$\begin{array}{l}\text { Fisher exact test or } \\
\text { studentt-test, -value }\end{array}$} \\
\hline & Mean & SD & Mean & SD & Mean & SD & \\
\hline Female gender, $\%$ & 40 & & 39 & & 39 & & 0.892 \\
\hline Age, y & 52.3 & 19.0 & 63.6 & 15.7 & 59.5 & 17.8 & $<0.001$ \\
\hline APACHE I| score & 21.7 & 8.6 & 24.4 & 7.7 & 23.4 & 8.1 & 0.013 \\
\hline Weight, kg & 80.2 & 15.6 & 73.6 & 15.1 & 76.0 & 15.6 & 0.001 \\
\hline Height, cm & 174.4 & 9.7 & 173.0 & 9.5 & 173.5 & 9.5 & 0.281 \\
\hline $\mathrm{BMl}, \mathrm{kg} / \mathrm{m}^{2}$ & 26.3 & 4.3 & 24.4 & 4.1 & 25.1 & 4.2 & 0.001 \\
\hline Muscle mass, kg & 22.0 & 4.2 & 16.6 & 3.6 & 18.6 & 4.6 & $<0.001$ \\
\hline Muscle index, $\mathrm{cm}^{2} / \mathrm{m}^{2}$ & 54.5 & 8.7 & 39.4 & 8.3 & 45.0 & 4.6 & $<0.001$ \\
\hline Muscle area, $\mathrm{cm}^{2}$ & 167.1 & 35.8 & 119.1 & 30.2 & 136.7 & 39.8 & $<0.001$ \\
\hline \multicolumn{8}{|l|}{ Admission diagnosis, \% } \\
\hline Cardiovascular & 5.7 & & 14.5 & & 11.2 & & 0.009 \\
\hline Metabolic/Renal & 3.4 & & 1.3 & & 2.1 & & $\left(x^{2}\right.$ test $)$ \\
\hline Neurologic & 5.7 & & 3.9 & & 4.6 & & \\
\hline Postresuscitation & 3.4 & & 2.0 & & 2.5 & & \\
\hline Postsurgery & 26.1 & & 34.2 & & 31.2 & & \\
\hline Respiratory insufficiency & 9.1 & & 15.8 & & 13.3 & & \\
\hline Sepsis & 4.5 & & 7.2 & & 6.2 & & \\
\hline Trauma & 35.2 & & 14.5 & & 22.1 & & \\
\hline Others & 6.8 & & 6.6 & & 6.7 & & \\
\hline Length of ventilation, $d$ (median; IQR) & 15 & $8-28$ & 13 & $8-23$ & 14 & $8-25$ & 0.387 \\
\hline ICU length of stay, d (median; IQR) & 17 & $9-30$ & 16 & $10-28$ & 16 & $9-28$ & 0.768 \\
\hline Hospital length of stay, $d$ (median; IQR) & 42 & $20-64$ & 41 & $24-66$ & 41 & $22-65$ & 0.930 \\
\hline ICU mortality, \% & 10.2 & & 23.7 & & 18.8 & & 0.010 \\
\hline 28-d mortality, \% & 5.7 & & 21.7 & & 15.8 & & 0.001 \\
\hline Hospital mortality, \% & 12.5 & & 38.2 & & 28.8 & & $<0.001$ \\
\hline Survivor analysis, $n$ & 77 & & 93 & & 170 & & \\
\hline To nursing home, \% & 11.7 & & 24.7 & & 18.8 & & 0.032 \\
\hline
\end{tabular}

APACHE, Acute Physiologic and Chronic Health Evaluation; BMI, Body mass index; IQR, interquartile range.

based on weight and height; however, weight includes both fat mass and muscle mass. For healthy obese females, we have shown that the mean value for fat-free mass increases from 40 to almost $60 \mathrm{~kg}$ between BMI 25 and BMI 50 [15]. Because heavier people carry a significant weight, they are likely to have an increased muscle mass along with an even much more increased fat mass. As a result, the assessment of $\mathrm{BMI}$, without any insight into body composition, does not prove that increased fat mass is beneficial for ICU patients. A significant problem may also be the limited accuracy of weight assessment in ICU patients. In a large group of almost 1,500 oncology patients, Martin et al. [22] showed that the poor prognosis based on weight loss, low muscle index, and muscle attenuation was independent of weight status (underweight, normal weight, overweight, and obesity).
In addition, it was shown before that sarcopenic obesity, being the combination of a high BMI and a low muscle mass, was related to a poor prognosis, in particular in cancer patients [12]. The study by Martin et al. extends the finding that low muscle index is prognostic for mortality, independent of weight status and not only for obese patients. Moisey et al. added the risk of low muscle for mortality in elderly injured ICU patients, while not showing a relation between fat tissue and mortality [20].

Previously we showed that reaching both protein and energy targets in critically ill patients had a positive impact on survival [23,24]. In an earlier analysis, we were able to show beneficial effects of optimal nutrition only in females [23]. Females by nature have a lower muscle mass and thus a lower body-protein mass as 


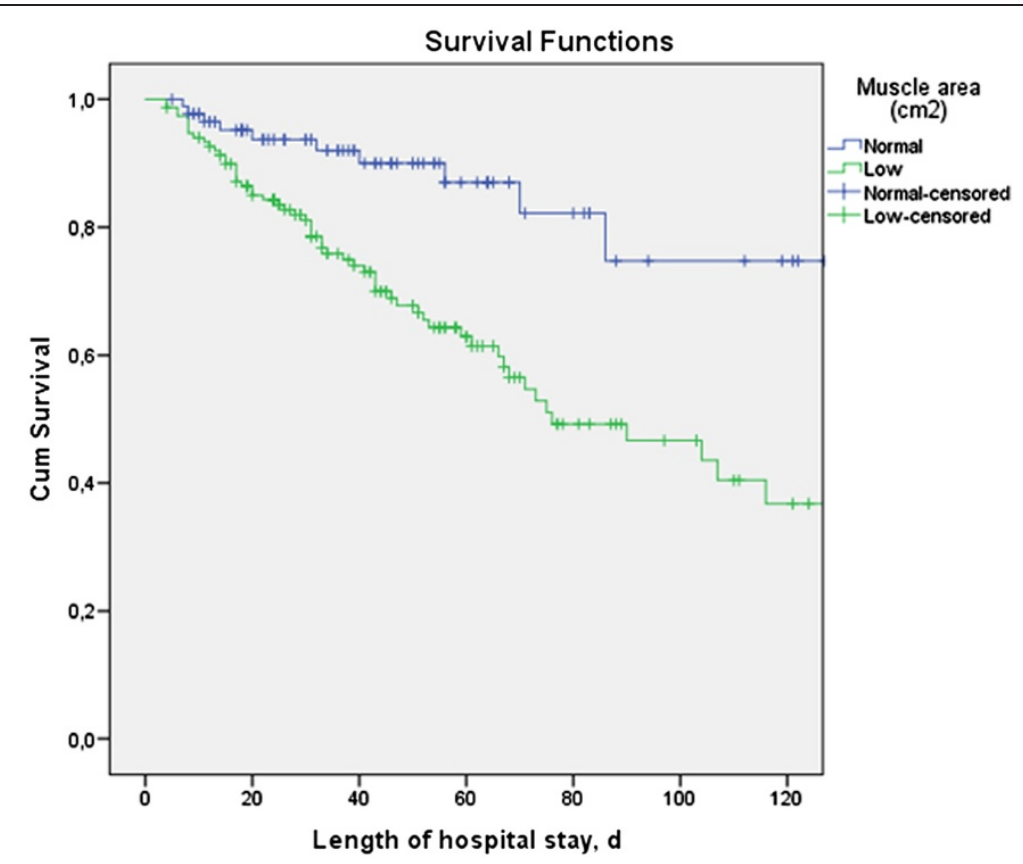

Figure 2 Kaplan-Meier survival plot for low- and normal-muscle area group (log rank test, $P<0.001$ ).

compared with males. We therefore hypothesized that the lower protein mass in females might be prone to reaching a critical point earlier under the stressed conditions of ICU admission. Our present study seems to confirm this hypothesis, because we found that females had a lower muscle mass than males. In addition, if muscle area was introduced as a continuous variable and not as a sex-related cut-off category, sex disappeared as independent predictor of mortality.
When we applied both sex-specific cutoffs to both females and males, this high-medium-low group in the backward stepwise regression analysis obviated sex as a predictor. Therefore, we stress that the muscle mass and mortality issue is primarily a mass effect and that the gender issue is considered secondary. The gender/ muscle mass relation also sheds new light on previous studies reporting that female patients have a higher mortality when developing nosocomial infections in the ICU

Table 2 Backward stepwise (Wald) regression analyses

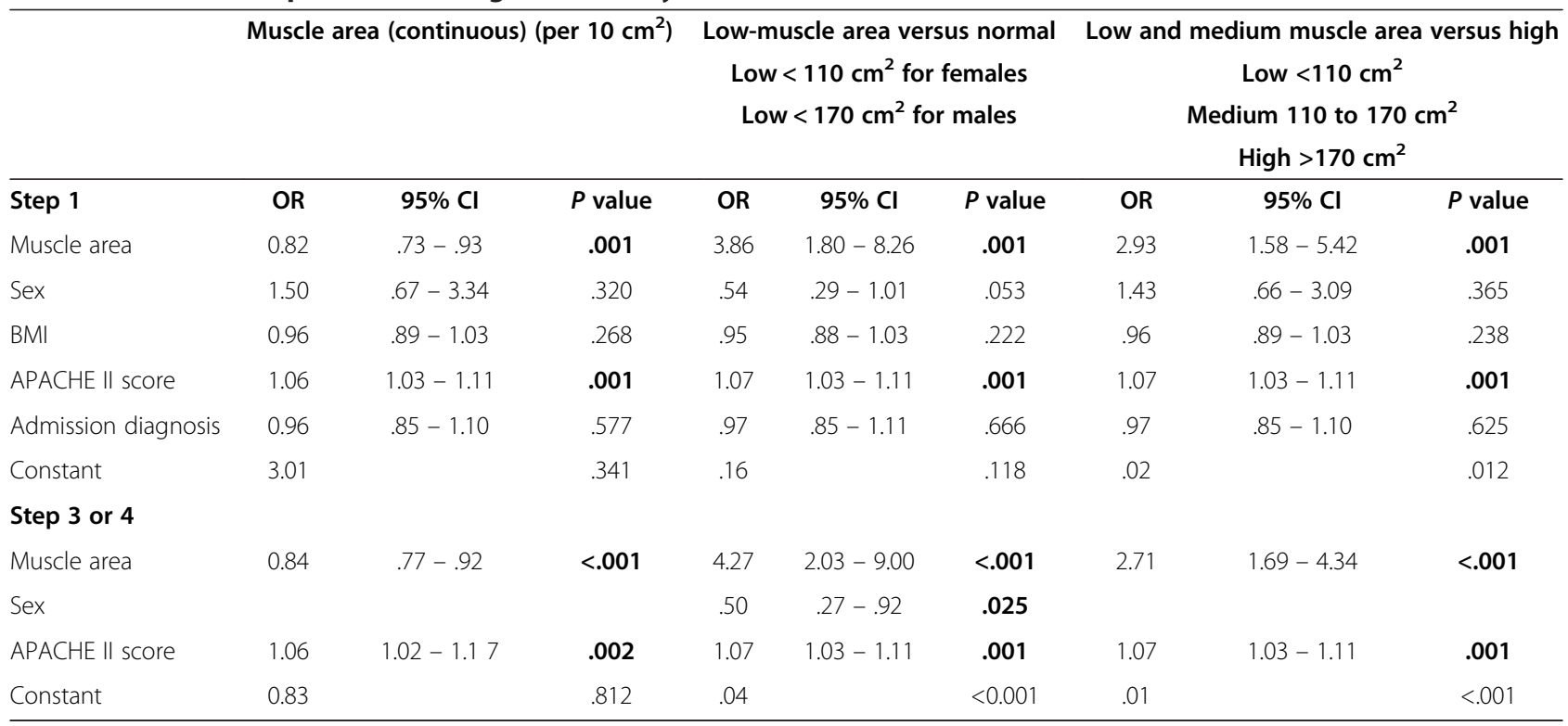


[25] or after cardiac surgery [26]. Reduced muscle mass in females may explain these observations and may prove to be an important denominator of poorer outcome.

The present study should be considered as a proof-ofconcept study. CT scans are an important tool to assess nutritional status of cancer patients, because scans are part of their routine monitoring. However, CT scans are not routinely performed in ICU patients and cannot be used as such because of X-ray exposure, lack of time, expertise, and high costs. Using CT scans for longitudinal monitoring is unfeasible, and for the intensive care population, alternatives for the CT scan should be explored. Caporossi et al. [27] assessed muscle mass by measuring thickness of the adductor pollicis muscle. Low thickness of this thumb muscle was associated with a 6 times higher risk of death in 246 ICU patients. In addition, low quadriceps femoris muscle mass, as assessed by ultrasound, was associated with longer ICU stay $[1,16]$. An advantage of using these approaches is that multiple longitudinal measures are easily obtained, and therefore allow long-term monitoring of muscle mass in the ICU patient $[28,29]$. An alternative option could be to monitor fat-free mass or phase angle by using bioelectrical impedance [30].

If the independent relation between low muscle mass and higher mortality is causative, the question arises whether therapeutic interventions can positively influence this relation. Possible interventions include optimization of nutrition, muscle training, or electrical muscle stimulation. The dosing of the nutritional protein component remains controversial. Most nutritional guidelines express protein needs per kilogram body weight, whereas only Ishibashi et al. [31] were able to advise 1.5-gram protein per $\mathrm{kg}$ fat-free mass. Low muscle mass is a likely indicator of undernutrition and/or poor mobility. If muscle mass could be assessed in daily clinical practice, this information could influence daily treatment [32]. Controversy exists about the timing of starting feeding in ICU patients. Early feeding could suppress autophagy, and protein seems the most important factor [33,34]. Monitoring muscle mass may at least contribute to the understanding of protein needs for ICU patients and provide a means to evaluate the effect and timing of different nutrition regimens.

The present study has strengths and limitations. It is the first study proving that measured muscle area by using novel techniques in critically ill patients is a predictor of patient outcome in a general ICU population, confirming the relation found in cancer patients and elderly injured patients. It also shows that muscle mass is a stronger predictor for outcome than BMI, suggesting that the patient's muscle reserve is more important than his fat reserve. Limitations include that it is unknown whether CT scan-derived muscle mass truly relates to total muscle mass in ICU patients. However, for healthy subjects [19] and cancer patients [13], a good relation has been shown. In addition, the timing of CT scanning was not standardized because scans performed for clinical reasons were used; this may have introduced some selection bias. Patients in whom a CT scan is made may not be representative for all ICU patients, which could be another source of selection bias. However, all scans were made within the first 4 days of ICU admission. Finally, CT scanning is not a feasible tool for routine monitoring of muscle mass, and alternative imaging tools such as ultrasound or bio-impedance should be explored.

\section{Conclusion}

Low skeletal muscle area is a risk factor for mortality in our cohort of mechanically ventilated critically ill patients who had a CT scan made during the first days of ICU admission. This observation was independent of sex and APACHE II score. Additional analysis suggested muscle mass as a primary predictor, not gender. Furthermore, when muscle area was accounted for, BMI was not a predictor of mortality. The ICU-specific cut-off values as found in our study need external validation.

\section{Key messages}

- Low skeletal muscle area is an independent risk factor for mortality in mechanically ventilated critically ill patients in an ICU representative age group.

- The effect of muscle is independent of sex and APACHE II score.

- When muscle area is accounted for, BMI appears to have no impact on mortality.

\section{Abbreviations}

APACHE: Acute Physiologic and Chronic Health Evaluation; BMI: body mass index; CT: computed tomography; ICU: intensive care unit; L3: third lumbar vertebra; LOS: length of stay; OR: odds ratio; PDMS: patient data-management system.

\section{Competing interests}

The authors declare that they have no competing interests.

\section{Authors' contributions}

$P W, A B, W L, H O$, and $A G$ designed the study. WL, AB, and SS obtained the data. WL and ID analyzed the CT scans. PW performed statistical analysis. All authors contributed in drafting the manuscript and authorized the final manuscript.

\section{Acknowledgements}

We thank Monique de Waard and Ronald Driessen from the Department of Intensive Care Medicine for their contributions. We also thank Vicky Baracos and Nina Esfandiari from University of Alberta, Canada, and Suzanne Blauwhoff-Buskermolen and Marian van Bokhorst from Nutrition and Dietetics for cooperation concerning the analysis of CT scans. 


\section{Author details}

${ }^{1}$ Department of Nutrition and Dietetics, Internal Medicine, VU University Medical Center Amsterdam, De Boelelaan 1117, Amsterdam, The Netherlands. ${ }^{2}$ Department of Intensive Care Medicine, VU University Medical Center Amsterdam, De Boelelaan 1117, Amsterdam, The Netherlands. ${ }^{3}$ Department of Nutrition and Dietetics, Amsterdam University of Applied Sciences, Dr. Meurerlaan 8, Amsterdam, The Netherlands. ${ }^{4}$ Department of Intensive Care Medicine, Medisch Spectrum Twente, Ariënsplein 1, Enschede, The Netherlands. ${ }^{5}$ Institute for Cardiovascular Research, VU University Medical Center Amsterdam, De Boelelaan 1117, Amsterdam, The Netherlands.

Received: 5 October 2013 Accepted: 3 January 2014

Published: 13 January 2014

\section{References}

1. Gruther W, Benesch T, Zorn C, Paternostro-Sluga T, Quittan M, Fialka-Moser $V$, Spiss C, Kainberger F, Crevenna R: Muscle wasting in intensive care patients: ultrasound observation of the $M$. quadriceps femoris muscle layer. J Rehabil Med 2008, 40:185-189.

2. Villet S, Chiolero RL, Bollmann MD, Revelly JP, Cayeux RN, Delarue J, Berger MM: Negative impact of hypocaloric feeding and energy balance on clinical outcome in ICU patients. Clin Nutr 2005, 24:502-509.

3. Dvir D, Cohen J, Singer P: Computerized energy balance and complications in critically ill patients: an observational study. Clin Nutr 2006, 25:37-44.

4. Oliveros $\mathrm{H}$, Villamor E: Obesity and mortality in critically ill adults: a systematic review and meta-analysis. Obesity (Silver Spring) 2008, 16:515-521

5. Hogue CWJ, Stearns JD, Colantuoni E, Robinson KA, Stierer T, Mitter N, Pronovost PJ, Needham DM: The impact of obesity on outcomes after critical illness: a meta-analysis. Intensive Care Med 2009, 35:1152-1170.

6. World Health Organization: Obesity: preventing and managing the global epidemic: report of a WHO consultation. World Health Organ Tech Rep Ser 2000, 894:i-253

7. Martino JL, Stapleton RD, Wang M, Day AG, Cahill NE, Dixon AE, Suratt BT, Heyland DK: Extreme obesity and outcomes in critically ill patients. Chest 2011, 140:1198-1206.

8. Sakr Y, Elia C, Mascia L, Barberis B, Cardellino S, Livigni S, Fiore G, Filippini C, Ranieri VM: Being overweight or obese is associated with decreased mortality in critically ill patients: a retrospective analysis of a large regional Italian multicenter cohort. J Crit Care 2012, 27:714-721.

9. Pickkers P, de Keizer N, Dusseljee J, Weerheijm D, van der Hoeven JG, Peek N: Body mass index is associated with hospital mortality in critically ill patients: an observational cohort study. Crit Care Med 2013, 41:1878-1883.

10. Kalantar-Zadeh K, Streja E, Molnar MZ, Lukowsky LR, Krishnan M, Kovesdy $C P$, Greenland S: Mortality prediction by surrogates of body composition: an examination of the obesity paradox in hemodialysis patients using composite ranking score analysis. Am J Epidemiol 2012, 175:793-803.

11. Mourtzakis M, Prado CMM, Lieffers JR, Reiman T, McCargar LJ, Baracos VE: A practical and precise approach to quantification of body composition in cancer patients using computed tomography images acquired during routine care. Appl Physiol Nutr Metab 2008, 33:997-1006.

12. Baracos VE, Reiman T, Mourtzakis M, Gioulbasanis I, Antoun S: Body composition in patients with non-small cell lung cancer: a contemporary view of cancer cachexia with the use of computed tomography image analysis. Am J Clin Nutr 2010, 91:1133S-1137S

13. Prado CMM, Lieffers JR, McCargar LJ, Reiman T, Sawyer MB, Martin L, Baracos VE: Prevalence and clinical implications of sarcopenic obesity in patients with solid tumours of the respiratory and gastrointestinal tracts: a population-based study. Lancet Oncol 2008, 9:629-635.

14. Thoresen L, Frykholm G, Lydersen S, Ulveland H, Baracos V, Prado CMM, Birdsell L, Falkmer U: Nutritional status, cachexia and survival in patients with advanced colorectal carcinoma: different assessment criteria for nutritional status provide unequal results. Clin Nutr 2013, 32:65-72.

15. Weijs PJM, Vansant GAAM: Validity of predictive equations for resting energy expenditure in Belgian normal weight to morbid obese women. Clin Nutr 2010, 29:347-351

16. Weijs PJM, Wischmeyer PE: Optimizing energy and protein balance in the ICU. Curr Opin Clin Nutr Metab Care 2013, 16:194-201.
17. Heymsfield SB, Wang Z, Baumgartner RN, Ross R: Human body composition: advances in models and methods. Annu Rev Nutr 1997, 17:527-558.

18. Mitsiopoulos N, Baumgartner RN, Heymsfield SB, Lyons W, Gallagher D, Ross R: Cadaver validation of skeletal muscle measurement by magnetic resonance imaging and computerized tomography. J App/ Physiol 1998, 85:115-122.

19. Shen W, Punyanitya M, Wang Z, Gallagher D, St-Onge MP, Albu J, Heymsfield SB, Heshka S: Total body skeletal muscle and adipose tissue volumes: estimation from a single abdominal cross-sectional image. J Appl Physiol 2004, 97:2333-2338.

20. Moisey L, Mourtzakis M, Cotton B, Premii T, Heyland D, Wade C, Bulger E, Kozar R: Skeletal muscle predicts ventilator-free days, ICU-free days, and mortality in elderly ICU patients. Crit Care 2013, 17:R206.

21. Kiraly L, Hurt RT, Van Way CW: The outcomes of obese patients in critical care. JPEN J Parenter Enteral Nutr 2011, 35:29S-35S

22. Martin L, Birdsell L, Macdonald N, Reiman T, Clandinin MT, McCargar L, Murphy R, Ghosh S, Sawyer MB, Baracos VE: Cancer cachexia in the age of obesity: skeletal muscle depletion is a powerful prognostic factor independent of body mass index. J Clin Oncol 2013, 31:1539-1547.

23. van Schijndel RJM S, Weijs PJM, Koopmans RH, Sauerwein HP, Beishuizen A, Girbes ARJ: Optimal nutrition during the period of mechanical ventilation decreases mortality in critically ill, long-term acute female patients: a prospective observational cohort study. Crit Care 2009, 13:R132.

24. Weijs PJM, Stapel SN, de Groot SDW, Driessen RH, de Jong E, Girbes ARJ, van Schijndel RJMS, Beishuizen A: Optimal protein and energy nutrition decreases mortality in mechanically ventilated, critically ill patients: a prospective observational cohort study. JPEN J Parenter Enteral Nutr 2012 36:60-68.

25. Combes A, Luyt CE, Trouillet JL, Nieszkowska A, Chastre J: Gender impact on the outcomes of critically ill patients with nosocomial infections. Crit Care Med 2009, 37:2506-2511.

26. Blankstein R, Ward RP, Arnsdorf M, Jones B, Lou YB, Pine M: Female gender is an independent predictor of operative mortality after coronary artery bypass graft surgery: contemporary analysis of 31 Midwestern hospitals. Circulation 2005, 112:1323-1327.

27. Caporossi FS, Caporossi C, Borges Dock-Nascimento D, de Aguilar-Nascimento JE: Measurement of the thickness of the adductor pollicis muscle as a predictor of outcome in critically ill patients. Nutr Hosp 2012, 27:490-495.

28. Puthucheary ZA, Rawal J, McPhail M, Connolly B, Ratnayake G, Chan P, Hopkinson NS, Padhke R, Dew T, Sidhu PS, Velloso C, Seymour J, Agley CC, Selby A, Limb M, Edwards LM, Smith K, Rowlerson A, Rennie MJ, Moxham J, Harridge SDR, Hart N, Montgomery HE: Acute skeletal muscle wasting in critical illness. JAMA 2013, 310:1591-1600.

29. Braunschweig C, Sheean P, Peterson S, Perez S, Freels S, Troy K, Ajanaku F, Patel A, Sclamberg J, Wang Z: Exploitation of diagnostic computed tomography scans to assess the impact of nutritional support on body composition changes in respiratory failure patients. JPEN J Parenter Enteral Nutr 2013. Epub ahead of print.

30. Visser M, van Venrooij LMW, Wanders DCM, de Vos R, Wisselink W, van Leeuwen PAM, de Mol BAJM: The bioelectrical impedance phase angle as an indicator of undernutrition and adverse clinical outcome in cardiac surgical patients. Clin Nutr 2012, 31:981-986

31. Ishibashi N, Plank LD, Sando K, Hill GL: Optimal protein requirements during the first 2 weeks after the onset of critical illness. Crit Care Med 1998, 26:1529-1535.

32. Weijs PJM, Sauerwein HP, Kondrup J: Protein recommendations in the ICU: $\mathrm{g}$ protein/kg body weight: which body weight for underweight and obese patients? Clin Nutr 2012, 31:774-775.

33. Rice TW, Wheeler AP, Thompson BT, Steingrub J, Hite RD, Moss M, Morris A, Dong N, Rock P: Initial trophic vs full enteral feeding in patients with acute lung injury: the EDEN randomized trial. JAMA 2012, 307:795-803.

34. Casaer MP, Hermans G, Wilmer A, Van den Berghe G: Impact of early parenteral nutrition completing enteral nutrition in adult critically il patients (EPaNIC trial): a study protocol and statistical analysis plan for a randomized controlled trial. Trials 2011, 12:21.

doi:10.1186/cc13189

Cite this article as: Weijs et al:: Low skeletal muscle area is a risk factor for mortality in mechanically ventilated critically ill patients. Critical Care 2014 18:R12. 